\title{
Article
}

\section{Balances in the Set of Arithmetic Progressions ${ }^{\dagger}$}

\author{
Chan-Liang Chung ${ }^{*} \ddagger \oplus$, Chunmei Zhong $\ddagger$ and Kanglun Zhou $\ddagger$
}

check for

updates

Citation: Chung, C.-L.; Zhong, C.; Zhou, K. Balances in the Set of Arithmetic Progressions. Axioms 2021, 10, 350. http://doi.org/10.3390/ axioms 10040350

Academic Editor: Takao Komatsu

Received: 2 November 2021

Accepted: 26 November 2021

Published: 20 December 2021

Publisher's Note: MDPI stays neutral with regard to jurisdictional claims in published maps and institutional affiliations.

Copyright: (c) 2021 by the authors. Licensee MDPI, Basel, Switzerland. This article is an open access article distributed under the terms and conditions of the Creative Commons Attribution (CC BY) license (https:// creativecommons.org/licenses/by/ $4.0 /)$.
School of Mathematics and Statistics, Fuzhou University, No.2 North Road of Wulongiiang,

Fuzhou 350108, China; zhongchunmei38@gmail.com (C.Z.); zhoukanglunz@163.com (K.Z.)

* Correspondence: andrechung@fzu.edu.cn

+ For Fish Leong. If we know what love is, it is because of you.

$\ddagger$ These authors contributed equally to this work.

Abstract: This article focuses on searching and classifying balancing numbers in a set of arithmetic progressions. The sufficient and necessary conditions for the existence of balancing numbers are presented. Moreover, explicit formulae of balancing numbers and various relations are included.

Keywords: balancing numbers; sequence balancing numbers; $(a, b)$-type balancing numbers; Pell equation

\section{Introduction}

An integer $n$ is called a balancing number if there exists another integer $r$, called a balancer, corresponding to $n$, such that the following Diophantine equation holds

$$
1+2+\cdots+(n-1)=(n+1)+(n+2)+\cdots+(n+r) .
$$

For example, 6 is a balancing number with balancer 2 . We allow 1 to be the first balancing number with the first balancer 0 . Let $B_{m}$ be the $m$-th balancing number and $R_{m}$ be the $m$-th balancer. Then we have that $\left\{B_{m}\right\}_{m \geq 1}=\{1,6,35,204, \ldots\}$ (OEIS: A001109) and $\left\{R_{m}\right\}_{m \geq 1}=\{0,2,14,84, \ldots\}$ (OEIS: A053141). The problem of determining all balancing numbers in the set of natural numbers leads to a second order linear recursive sequence or a Pell equation. In [1], Behera and Panda prove that the square of any balancing number is a triangular number. In fact, it is easy to derive that $n$ is a balancing number if and only if $n^{2}$ is a triangular number, and if and only if $8 n^{2}+1$ is a perfect square. They also proved that the balancing sequence $\left\{B_{m}\right\}_{m \geq 1}$ fulfilled the recursive relation

$$
B_{m+1}=6 B_{m}-B_{m-1},
$$

for all $m \geq 2$ with initials $B_{1}=1, B_{2}=6$. According to the recursive relation, it forces that $B_{0}=0$.

From (1), we have

$$
r^{2}+(2 n+1) r-n(n-1)=0,
$$

or

$$
r=\frac{-2 n-1+\sqrt{8 n^{2}+1}}{2}
$$

Since $r$ is an integer, then $8 n^{2}+1$ must be an odd square, say $8 n^{2}+1=t^{2}$ with $t$ odd. We have

$$
n^{2}=\frac{\left(\frac{t-1}{2}\right)\left(\frac{t+1}{2}\right)}{2}=T_{(t-1) / 2}
$$

where $T_{m}:=m(m+1) / 2$ is the $m$-th triangular number. The $m$-th Lucas-balancing number may be defined by $C_{m}:=\sqrt{8 B_{m}^{2}+1}$ and $\left\{C_{m}\right\}_{m \geq 0}=\{1,3,17,99,577, \ldots\}$ (OEIS: A001541). 
In addition, one can check that $C_{m+1}=6 C_{m}-C_{m-1}$ and the relation $R_{m}=\frac{C_{m}-2 B_{m}-1}{2}$ for any $m \geq 1$.

Similarly, one can define the cobalancing numbers with cobalancers as the solutions in $n, r$ to the Diophantine equation

$$
1+2+\cdots+n=(n+1)+(n+2)+\cdots+(n+r) .
$$

From (2), it implies that $n$ is a cobalancing number if and only if $n(n+1)$ is a triangular number, and if and only if $8 n^{2}+8 n+1$ is a perfect square. Throughout this paper, let $b_{m}, r_{m}$ and $c_{m}:=\sqrt{8 b_{m}^{2}+8 b_{m}+1}$ be the $m$-th cobalancing number, the $m$-th cobalancer, and the $m$-th Lucas-balancing number, respectively. For example, $b_{1}=r_{1}=0$ and $c_{1}=1$.

Various possible generalizations of balancing numbers, balancers, and Lucas-balancing numbers have been studied by several authors from many aspects. In [2], Panda defined sequence balancing numbers with sequence balancers as follows. Let $\left\{u_{m}\right\}_{m \geq 1}$ be a sequence of real numbers. We call a pair $\left(u_{m}, k\right)$ a sequence balancing number with sequence balancer if

$$
u_{1}+u_{2}+\cdots+u_{m-1}=u_{m+1}+u_{m+2}+\cdots+u_{m+k} .
$$

Kovács, Liptai and Olajos [3] introduced the concept of sequence balancing numbers to the sequence of arithmetic progressions. They defined the $(a, b)$-type balancing numbers with the $(a, b)$-type balancer as solutions of the Diophantine equation

$$
(a+b)+(2 a+b)+\cdots+(a(n-1)+b)=(a(n+1)+b)+\cdots+(a(n+r)+b),
$$

where $a>0$ and $b \geq 0$ are coprime integers. In [3], several effective finiteness and explicit results about $(a, b)$-type balancing numbers had been given. In particular, Kovács et al. proved the following theorem.

Theorem 1 (Theorem 2 in [3]). If $a^{2}-4 a b-4 b^{2}=1$, then there is no perfect power $(a, b)$-type balancing number.

Thus, a question naturally arises: can we determine all $(a, b)$-type balancing numbers in the set of arithmetic progressions? In this paper, we will provide an answer to this question. In other words, we search "few" balances in the set of arithmetic progressions. First, we must be clear about the terminology and notation. We denote the $m$-th $(a, b)$-type balancing number, if it exists for infinitely many $m$, by $B_{m}^{(a, b)}$. Note that when $(a, b)=(1,0)$, we get nothing but the original balancing number $B_{m}$. Instead of requesting integers $a>0$ and $b \geq 0$, our definition of $(a, b)$-type balancing should only exclude from the cases $a=0$ $\operatorname{or} \operatorname{gcd}(a, b) \neq 1$. For the former case $a=0$, it is trivial. For the later case, we just notice for any integer $d \neq 0$ that

$$
B_{m}^{(d a, d b)}=d B_{m}^{(a, b)} .
$$

Without loss of generality, we may assume that $a>0$, for otherwise, use the above identity by putting $d=-1$. For our convenience, let $B_{0}^{(a, b)}=b$. We allow that 1 is the first balancing number, i.e., $B_{1}=1$, as well as $B_{1}^{(a, b)}=a+b$. The $(a, b)$-type balancer is defined to be the least number $r$, which satisfies (3) and the $m$-th $(a, b)$-type balancer is denoted by $R_{m}^{(a, b)}$ if it exists.

This paper is organized as follows. In Section 2, we present a sufficient and necessary condition for the existence of the $(a, b)$-type balancing number $B_{m}^{(a, b)}$ for infinitely many $m$. Precisely, we show numbers $B_{m}^{(a, b)}$ exist for infinitely many $m$ if and only if $a \mid 2 b$. Moreover, we sort and classify all $(a, b)$-type balancing numbers and provide explicit formulae for them. In Section 3, we discuss the $(a, b)$-type cobalancing numbers, the $(a, b)$ - 
type cobalancers, and the $(a, b)$-type Lucas-cobalancing numbers. We summarize the paper in the conclusion section.

\section{Main Results}

The problem of determining all $(a, b)$-type balancing numbers in the set of arithmetic progressions leads to the equation $x^{2}-8 y^{2}=c$. In the following, we derive the condition $a \mid 2 b$ as a sufficient and necessary condition for the existence of the $(a, b)$-type balancing number $B_{m}^{(a, b)}$ for infinitely many $m$.

On one hand, from (3) we have

$$
\frac{n(n-1)}{2} a+(n-1) b=a r n+\frac{r(r+1)}{2} a+b r,
$$

or

$$
a r^{2}+(2 a n+a+2 b) r-(n-1)(a n+2 b)=0 .
$$

We solve the equation in $r$ directly to get

$$
r=-n+\frac{-a-2 b \pm \sqrt{8(a n+b)^{2}+\left(a^{2}-4 a b-4 b^{2}\right)}}{2 a} .
$$

It "forces" that the number $8(a n+b)^{2}+\left(a^{2}-4 a b-4 b^{2}\right)$ must be a perfect square. We then define the $m$-th $(a, b)$-type Lucas-balancing number by

$$
C_{m}^{(a, b)}:=\sqrt{8\left(B_{m}^{(a, b)}\right)^{2}+a^{2}-4 a b-4 b^{2}} .
$$

Note that both $a$ and $C_{m}^{(a, b)}$ have the same parity. Now we have, for all $m \geq 1$,

$$
\left(C_{m}^{(a, b)}\right)^{2}-8\left(B_{m}^{(a, b)}\right)^{2}=a^{2}-4 a b-4 b^{2} .
$$

On the other hand, multiplying Equation (4) by $4 a$ (note that $a \neq 0$ ), we get

$$
\begin{aligned}
(2(a n+b)+a(2 r+1))^{2}-8(a n+b)^{2} & =a^{2}-4 a b-4 b^{2} \\
& =( \pm(a-2 b))^{2}-8 b^{2} .
\end{aligned}
$$

Let $x=2 a(n+r)+a+2 b$ and $y=a n+b$. We may rewrite (5) as

$$
x^{2}-8 y^{2}=c,
$$

where $c=a^{2}-4 a b-4 b^{2}=( \pm(a-2 b))^{2}-8 b^{2}$. To solve Equation (6), we solve the following Pell equation in two variables $u, v$ :

$$
u^{2}-8 v^{2}=1 .
$$

The fundamental solution is $(u, v)=(3,1)$ and, thus, all the solutions can be determined by

$$
u+v \sqrt{8}=(3+\sqrt{8})^{m},
$$

for any $m \geq 0$. Notice that $(x, y)=( \pm(a-2 b), b)$ is an initial solution of the Equation (6). Hence, all solutions of the Equation (6) takes the form

$$
x+y \sqrt{8}=( \pm(a-2 b)+b \sqrt{8})(3+\sqrt{8})^{m} .
$$


Suppose that $(3+\sqrt{8})^{m}=x_{m}+y_{m} \sqrt{8}$. It is easy to prove that both sequences $\left\{x_{m}\right\}_{m \geq 1},\left\{y_{m}\right\}_{m \geq 1}$ satisfy the same recurrence relation as below

$$
x_{m+1}=6 x_{m}-x_{m-1},
$$

for $m \geq 2$ and with initials $x_{1}=3, x_{2}=17$ and $y_{1}=1, y_{2}=6$, respectively. Therefore, we conclude that $x_{m}=C_{m}$ and $y_{m}=B_{m}$, the $m$-th Lucas-balancing number, and the $m$-th balancing number.

Now, we get

$$
x+y \sqrt{8}=( \pm(a-2 b)+b \sqrt{8})\left(C_{m}+B_{m} \sqrt{8}\right) .
$$

It immediately implies that

$$
B_{m}^{(a, b)}=b C_{m} \pm(a-2 b) B_{m} .
$$

Whether the positive or negative sign it takes, the key to guarantee is $B_{m}^{(a, b)} \geq B_{1}^{(a, b)}=a+b$. In light of $C_{m}=3 B_{m}-B_{m-1}$ (this follows easily by strong induction on $m$ ), we may rewrite the explicit formulae for the $(a, b)$-type balancing number as

$$
\begin{aligned}
& B_{m}^{(a, b)}=(a+b) B_{m}-b B_{m-1} ; \\
& B_{m}^{(a, b)}=b B_{m+1}-(a+b) B_{m} .
\end{aligned}
$$

For example, when $(a, b)=(2,1)$, we have $B_{m}^{(2,1)}=C_{m}=3 B_{m}-B_{m-1}$ for all $m \geq 1$. Another example, when $a=b=1$, we relabel the $(1,1)$-type balancing numbers according to Equation (7). That is, for $k \geq 1$,

$$
B_{0}^{(1,1)}=1, \begin{aligned}
& B_{2 k-1}^{(1,1)}=2 B_{k}-B_{k-1} \\
& B_{2 k}^{(1,1)}=B_{k+1}-2 B_{k} .
\end{aligned}
$$

Indeed, we have $\left\{B_{m}^{(1,1)}\right\}_{m \geq 0}=\{1,2,4,11,23,64,134,373,781, \ldots\}$ (OEIS:A006452) and that

$$
B_{m}^{(1,1)}=6 B_{m-2}^{(1,1)}-B_{m-4^{\prime}}^{(1,1)} \quad \text { for } m \geq 4 .
$$

Actually the number $B_{m}^{(1,1)}$ is an integer $n$, such that $8 n^{2}-7$ is a perfect square. Thus, $n^{2}-1$ is a triangular number. In [4], Subramaniam studied such an integer $n$, which he called the almost square triangular number (ASTN) of type $\beta$, such that $n^{2}-1$ is triangular. See also Theorem 2.2.2 in [2].

In addition, we see that the $x$-solutions of Equation (5) are given by

$$
\begin{aligned}
& 2 B_{m}^{(a, b)}+a\left(2 R_{m}^{(a, b)}+1\right)=8 b B_{m} \pm(a-2 b) C_{m}, \\
& C_{m}^{(a, b)}=\left|2 B_{m}^{(a, b)}+a\left(2 R_{m}^{(a, b)}+1\right)\right| \geq 0 .
\end{aligned}
$$

In light of $B_{m}^{(a, b)}=b C_{m} \pm(a-2 b) B_{m}$ and $R_{m}=\frac{C_{m}-2 B_{m}-1}{2}$, we can solve for $R_{m}^{(a, b)}$ from (8):

$$
\left\{\begin{array}{l}
R_{m}^{(a, b)}=\left(\frac{6 b}{a}-1\right) B_{m}-\frac{2 b}{a} C_{m}+\frac{C_{m}-1}{2}=\frac{2 b}{a} B_{m-1}+R_{m} \\
R_{m}^{(a, b)}=\left(1+\frac{2 b}{a}\right) B_{m}-\frac{C_{m}+1}{2}=\frac{2 b}{a} B_{m}-R_{m}-1,
\end{array}\right.
$$

and the recursive relation $R_{m+1}^{(a, b)}=6 R_{m}^{(a, b)}-R_{m-1}^{(a, b)}+2$ for all $m \geq 2$. 
Recall that $8 n^{2}+1$ is an odd square if $n$ is a balancing number. Thus, the number $C_{m}$ must be an odd integer for each positive integer $m$. Hence, we conclude that

$$
R_{m}^{(a, b)} \in \mathbb{Z} \Longleftrightarrow a \mid 2 b .
$$

That is, under the restriction of $\operatorname{gcd}(a, b)=1$, the positive integer $a$ is taken either 1 or 2 . We summarize the above discussion in the following theorem.

Theorem 2. Let $a, b$ be two coprime integers with $a \neq 0$. The sufficient and necessary condition for the existence of the $(a, b)$-type balancing number $B_{m}^{(a, b)}$, the $(a, b)$-type balancer $R_{m}^{(a, b)}$, and the $(a, b)$-type Lucas-balancing number $C_{m}^{(a, b)}$ for all $m \geq 1$ is $a \mid 2 b$.

We now give explicit formulae for the $(a, b)$-type balancing numbers with the $(a, b)$ type balancers by distinguishing four cases, according to $a=1$ or $a=2$.

(Case 1: $(a, b)=(1, b), b \geq 0$.) If $b=0$, we get exactly the original balancing number and balancer, $B_{m}^{(1,0)}=B_{m}, R_{m}^{(1,0)}=R_{m}$. For $b \geq 1$, in this case, the two relations in (7) make sense, since both $B_{m}^{(a, b)} \geq 1+b$ for all $m$. Hence, we just relabel and conclude that the explicit formula for the $(1, b)$-type balancing numbers is given by, for all $k \geq 1$,

$$
B_{0}^{(1, b)}=b, \quad\left\{\begin{array}{l}
B_{2 k-1}^{(1, b)}=(b+1) B_{k}-b B_{k-1} \\
B_{2 k}^{(1, b)}=b B_{k+1}-(b+1) B_{k} .
\end{array}\right.
$$

In light of (8) and (9), the corresponding $(1, b)$-type balancers $R_{m}^{(1, b)}$ and Lucas-balancing numbers $C_{m}^{(1, b)}$ are then given by

$$
\left\{\begin{array}{l}
R_{2 k-1}^{(1, b)}=2 b B_{k-1}+R_{k}, \quad C_{2 k-1}^{(1, b)}=8 b B_{k}+(1-2 b) C_{k} \\
R_{2 k}^{(1, b)}=2 b B_{k}-R_{k}-1, \quad C_{2 k}^{(1, b)}=8 b B_{k}-(1-2 b) C_{k}
\end{array}\right.
$$

for all $k \geq 1$.

(Case 2: $(a, b)=(1,-c), c \geq 1$.) In this case, we search the balances in the sequence $\{n-c\}_{n \geq 1}=\{1-c, 2-c, \ldots,-1,0,1, \ldots, c-1, c, c+1, \ldots\}$. By definition, $B_{1}^{(1,-c)}=1-c$. We see that the first few elements of the sequence $\{n-c\}_{n \geq 1}$ are negative, and they must cancel each other out with the first few positive elements. In fact, if $c \notin\left\{B_{\ell}^{(1, t)}+R_{\ell}^{(1, t)}+1\right\}_{t \geq 0, \ell \geq 2}$, then it is easy to see that $B_{m}^{(1,-c)}=B_{m}^{(1, c-1)}$ for all $m \geq 2$. If $c=B_{\ell}^{(1, t)}+R_{\ell}^{(1, t)}+1$ for some $t \geq 0$ and for some $\ell \geq 2$, then $B_{2}^{(1,-c)}=-B_{\ell}^{(1, t)}$ and $B_{m}^{(1,-c)}=B_{m-1}^{(1, c-1)}$ for all $m \geq 3$. To see this, just notice the identity

$$
\begin{aligned}
& \left(-B_{\ell}^{(1, t)}-R_{\ell}^{(1, t)}\right)+\left(-B_{\ell}^{(1, t)}-R_{\ell}^{(1, t)}+1\right)+\cdots+\left(-B_{\ell}^{(1, t)}-1\right) \\
= & \left(-B_{\ell}^{(1, t)}+1\right)+\left(-B_{\ell}^{(1, t)}+2\right)+\cdots+(t+2)+(t+1) .
\end{aligned}
$$

The above identity holds by the definition of balancing numbers with the balancer.

The corresponding $(1,-c)$-type balancers $R_{m}^{(1,-c)}$ are given as below. By definition, $R_{1}^{(1,-c)}=0$. Moreover, we have

$$
R_{2}^{(1,-c)}= \begin{cases}2, & \text { if } c=1 \\ R_{2}^{(1, c-1)}=2 c-3, & \text { if } c>1 \text { and } c \notin\left\{B_{\ell}^{(1, t)}+R_{\ell}^{(1, t)}+1\right\}_{t \geq 0, \ell \geq 2} \\ B_{\ell}^{(1, t)}-t-1, & \text { if } c=B_{\ell}^{(1, t)}+R_{\ell}^{(1, t)}+1 \text { for some } t \geq 0, \ell \geq 2\end{cases}
$$

and for $m \geq 3, R_{m}^{(1,-c)}=R_{m}^{(1, c-1)}$ if $c \notin\left\{B_{\ell}^{(1, t)}+R_{\ell}^{(1, t)}+1\right\}_{t \geq 0, \ell \geq 2}$ and $R_{m}^{(1,-c)}=R_{m-1}^{(1, c-1)}$ for otherwise. 
In addition, we have that $C_{1}^{(1,-c)}=|3-2 c|$ and

$$
C_{2}^{(1,-c)}= \begin{cases}17, & \text { if } c=1 \\ 14 c-17, & \text { if } c>1 \text { and } c \notin\left\{B_{\ell}^{(1, t)}+R_{\ell}^{(1, t)}+1\right\}_{t \geq 0, \ell \geq 2} \\ 2 t+1, & \text { if } c=B_{\ell}^{(1, t)}+R_{\ell}^{(1, t)}+1 \text { for some } t \geq 0, \ell \geq 2\end{cases}
$$

and for $m \geq 3$,

$$
C_{m}^{(1,-c)}= \begin{cases}2 B_{m}^{(1, c-1)}+2 R_{m}^{(1, c-1)}+1, & \text { if } c \notin\left\{B_{\ell}^{(1, t)}+R_{\ell}^{(1, t)}+1\right\}_{t \geq 0, \ell \geq 2} ; \\ 2 B_{m-1}^{(1, c-1)}+2 R_{m-1}^{(1, c-1)}+1, & \text { if } c=B_{\ell}^{(1, t)}+R_{\ell}^{(1, t)}+1 \text { for some } t \geq 0, \ell \geq 2 .\end{cases}
$$

(Case 3: $(a, b)=(2, b), b \geq 1$ odd.) When $(a, b)=(2,1)$, we obtain that $B_{m}^{(2,1)}=3 B_{m}-B_{m-1}$ by (7). Thus, it implies that $B_{m}^{(2,1)}=C_{m}$. So the balancing numbers in the sequence $\{3,5,7, \ldots\}$ are those Lucas-balancing numbers in the sequence of positive integers. Moreover, by (8) and (9), $R_{m}^{(2,1)}=B_{m-1}+R_{m}$ and $C_{m}^{(2,1)}=2\left(B_{m}+B_{m-1}+C_{m}\right)$.

For $b \geq 3$ is an odd integer, we have $B_{0}^{(2, b)}=b$ and for all $k \geq 1$

$$
\left\{\begin{array}{l}
B_{2 k-1}^{(2, b)}=(b+2) B_{k}-b B_{k-1} \\
B_{2 k}^{(2, b)}=b B_{k+1}-(b+2) B_{k}
\end{array}\right.
$$

In viewing of (8) and (9), the corresponding $(2, b)$-type balancers $R_{m}^{(2, b)}$ and Lucas-balancing numbers $C_{m}^{(2, b)}$ are then given by

$$
\left\{\begin{array}{l}
R_{2 k-1}^{(2, b)}=b B_{k-1}+R_{k}, \quad C_{2 k-1}^{(2, b)}=8 b B_{k}+2(1-b) C_{k} \\
R_{2 k}^{(2, b)}=b B_{k}-R_{k}-1, \quad C_{2 k}^{(2, b)}=8 b B_{k}-2(1-b) C_{k}
\end{array}\right.
$$

for all $k \geq 1$.

(Case 4: $(a, b)=(2,-c), c \geq 1$ odd.) The simplest subcase $c=1$ gives $B_{m}^{(2,-1)}=$ $B_{m}+B_{m-1}$. The sequence of integers $\left\{B_{m}^{(2,-1)}\right\}_{m \geq 1}$ begins with $1,7,41,239,1393,8119, \ldots$, which are related to the square order of simple groups and these numbers are called NSW numbers [5]. The NSW numbers have nice properties similar to Mersenne numbers $M_{p}=2^{p}-1$ with $p$ prime. It is easy to see that NSW numbers verify the recurrence relation $B_{m+1}^{(2,-1)}=6 B_{m}^{(2,-1)}-B_{m-1}^{(2,-1)}$. E. Barcucci et al. [6] gave a combinatorial interpretation of the sequence of NSW numbers. Actually, they proved that the cardinality of the set of words of $\mathfrak{L}$ having length $m$ is equal to $B_{m+1}^{(2,1)}$.

For all $m \geq 1$, the corresponding $(2,-1)$-type balancers $R_{m}^{(2,-1)}$ and Lucas-balancing numbers $C_{m}^{(2,-1)}$ are given by

$$
R_{m}^{(2,-1)}=R_{m}-B_{m-1}, C_{m}^{(2,-1)}=4\left(C_{m}-2 B_{m}\right)=4\left(B_{m}-B_{m-1}\right),
$$

respectively.

For $c>1$ is an odd integer, we note that $B_{1}^{(2,-c)}=2-c$. The identity

$$
\begin{aligned}
& \left(-B_{\ell}^{(2,2 t-1)}-2 R_{\ell}^{(2,2 t-1)}\right)+\left(-B_{\ell}^{(2,2 t-1)}-2 R_{\ell}^{(2,2 t-1)}+2\right)+\cdots+\left(-B_{\ell}^{(2,2 t-1)}-2\right) \\
= & \left(-B_{\ell}^{(2,2 t-1)}+2\right)+\cdots+(2 t-5)+(2 t-3)
\end{aligned}
$$


implies that $B_{2}^{(2,-c)}=-B_{\ell}^{(2,2 t-1)}$ if $c=B_{\ell}^{(2,2 t-1)}+2 R_{\ell}^{(2,2 t-1)}+2$ for some $t \geq 0$ and for some $\ell \geq 2$. If $c \notin\left\{B_{\ell}^{(2,2 t-1)}+2 R_{\ell}^{(2,2 t-1)}+2\right\}_{t \geq 0, \ell \geq 2}$, we have that $B_{2}^{(2,-c)}=B_{2}^{(2, c-2)}$. For $m \geq 3$, we have

$$
\begin{cases}B_{m}^{(2,-c)}=B_{m}^{(2, c-2)} & \text { if } c \notin\left\{B_{\ell}^{(2,2 t-1)}+2 R_{\ell}^{(2,2 t-1)}+2\right\}_{t \geq 0, \ell \geq 2} \\ B_{m}^{(2,-c)}=B_{m-1}^{(2, c-2)} & \text { for otherwise. }\end{cases}
$$

The corresponding $(2,-c)$-type balancers $R_{m}^{(2,-c)}$ are given as below. By definition, $R_{1}^{(2,-c)}=0$. Moreover, we have

$$
R_{2}^{(2,-c)}= \begin{cases}3 & \text { if } c=3 \\ R_{2}^{(2, c-2)}=c-3, & \text { if } c>3 \text { odd and } c \notin\left\{B_{\ell}^{(2,2 t-1)}+R_{\ell}^{(2,2 t-1)}+2\right\}_{t \geq 0, \ell \geq 2} ; \\ \frac{B_{\ell}^{(2,2 t-1)}+2 t-3}{2}, & \text { if } c=B_{\ell}^{(2,2 t-1)}+R_{\ell}^{(2,2 t-1)}+2 \text { for some } t \geq 0, \ell \geq 2,\end{cases}
$$

and for $m \geq 3, R_{m}^{(2,-c)}=R_{m}^{(2, c-2)}$ if $c \notin\left\{B_{\ell}^{(2,2 t-1)}+R_{\ell}^{(2,2 t-1)}+2\right\}_{t \geq 0, \ell \geq 2}$ and $R_{m}^{(2,-c)}=$ $R_{m-1}^{(2, c-2)}$ for otherwise.

Furthermore, we have that $C_{1}^{(2,-c)}=|6-2 c|$ for $c>1$ odd and

$$
C_{2}^{(2,-c)}= \begin{cases}48, & \text { if } c=3 ; \\ 14 c-34, & \text { if } c>3 \text { odd and } c \notin\left\{B_{\ell}^{(2,2 t-1)}+R_{\ell}^{(2,2 t-1)}+2\right\}_{t \geq 0, \ell \geq 2} \\ |4 t-4|, & \text { if } c=B_{\ell}^{(2,2 t-1)}+R_{\ell}^{(2,2 t-1)}+2 \text { for some } t \geq 0, \ell \geq 2\end{cases}
$$

and for $m \geq 3, C_{m}^{(2,-c)}=2 B_{m}^{(2, c-2)}+4 R_{m}^{(2, c-2)}+2$ if $c \notin\left\{B_{\ell}^{(2,2 t-1)}+R_{\ell}^{(2,2 t-1)}+2\right\}_{t \geq 0, \ell \geq 2}$ and $C_{m}^{(2,-c)}=2 B_{m-1}^{(2, c-2)}+4 R_{m-1}^{(2, c-2)}+2$ for otherwise.

\section{3. $(a, b)$-Type Cobalancing Numbers}

Let $a, b$ be two coprime integers with $a>0$. We say that $a n+b$ is an $(a, b)$-type cobalancing number if

$$
(a+b)+(2 a+b)+\cdots+(a n+b)=(a(n+1)+b)+\cdots+(a(n+r)+b),
$$

with a corresponding number $r>0$, which is called the cobalancer. Let $b_{m}^{(a, b)}$ and $r_{m}^{(a, b)}$ be the $m$-th $(a, b)$-type cobalancing number, the $m$-th $(a, b)$-type cobalancer, respectively.

The Equation (10) is equivalent to

$$
(2(a n+b)+a(1-2 r))^{2}-8(a r)^{2}=(a+2 b)^{2},
$$

or $x^{2}-8 y^{2}=c$ with $x=2(a n+b)+a(1-2 r), y=a r$ and $c=(a+2 b)^{2}$. If $c=0$; that is $a=-2 b$, the equation has no nonzero solution. For $c \neq 0$, all integral solutions can be determined by

$$
x+y \sqrt{8}=(a+2 b)(3+\sqrt{8})^{m}=(a+2 b)\left(C_{m}+B_{m} \sqrt{8}\right),
$$

for any $m \geq 0$. It implies that

$$
a r_{m}^{(a, b)}=(a+2 b) B_{m-1}=(a+2 b) r_{m}
$$

and

$$
b_{m}^{(a, b)}=b C_{m-1}+a \frac{C_{m-1}-1}{2}+(a+2 b) B_{m-1} .
$$

That is, $r_{m}^{(a, b)}=\left(1+\frac{2 b}{a}\right) r_{m}$ and it implies that $r_{m}^{(a, b)} \in \mathbb{Z}$ if and only if $a \mid 2 b$. 
If we solve the Equation (11) in $r$ directly, we obtain

$$
r=-n+\frac{-a-2 b \pm \sqrt{8(a n+b)^{2}+8 a(a n+b)+a^{2}-4 a b-4 b^{2}}}{2 a} .
$$

It forces that the number $8(a n+b)^{2}+8 a(a n+b)+a^{2}-4 a b-4 b^{2}$ must be a perfect square number. Thus, we define the $m$-th $(a, b)$-type Lucas-cobalancing number by $c_{m}^{(a, b)}:=\sqrt{8\left(b_{m}^{(a, b)}\right)^{2}+8 a b_{m}^{(a, b)}+a^{2}-4 a b-4 b^{2}}$. In other words, for all $m \geq 1$,

$$
\left(c_{m}^{(a, b)}\right)^{2}-2\left(2 b_{m}^{(a, b)}+a\right)^{2}=(a+2 b)^{2}-2(a+2 b)^{2} .
$$

Hence, the integral solutions can be determined by

$$
c_{m}^{(a, b)}+\left(2 b_{m}^{(a, b)}+a\right) \sqrt{2}=(a+2 b)(1+\sqrt{2})(3+2 \sqrt{2})^{m-1}
$$

for any $m \geq 1$. Then, from (13) and (14), we deduce the identity (12) and also

$$
c_{m}^{(a, b)}=(a+2 b)\left(C_{m-1}+4 B_{m-1}\right) .
$$

Notice that when $(a, b)=(2,-1)$, in this case, we have $a=-2 b$, then there do not exist any $(2,-1)$-type cobalancing numbers (in the sequence of odd positive integers) and $c_{m}^{(2,-1)}=0$ for all $m \geq 1$. This result coincides with Theorem 2.1.6 given in [2].

According to the identity (12), the following theorem follows easily by induction on $m$.

Theorem 3. Let $a, b$ be two coprime integers with $a>0$, and $a \mid 2 b$. Then we have for $m \geq 1$

$$
b_{m+1}^{(a, b)}=6 b_{m}^{(a, b)}-b_{m-1}^{(a, b)}+2 a,
$$

with initial values $b_{0}^{(a, b)}=b_{1}^{(a, b)}=b$. Moreover, for $m \geq 2$, we have

$$
r_{m+1}^{(a, b)}=6 r_{m}^{(a, b)}-r_{m-1}^{(a, b)}, \quad c_{m+1}^{(a, b)}=6 c_{m}^{(a, b)}-c_{m-1}^{(a, b)},
$$

with initials $r_{1}^{(a, b)}=0, r_{2}^{(a, b)}=\left(1+\frac{2 b}{a}\right)$, and $c_{1}^{(a, b)}=a+2 b, c_{2}^{(a, b)}=7 a+14 b$.

\section{Conclusions}

Our Theorem 2 says that the only guarantee of having the existence of $(a, b)$-type balancing numbers $B_{m}^{(a, b)}$ for infinitely many $m$ is by satisfying the condition $a \mid 2 b$. Although the equation $a^{2}-4 a b-4 b^{2}=1$ has infinitely many solutions in integers $a>0, b \geq 0$, it reduces the only one $(a, b)=(1,0)$ under the conditions $a \mid 2 b$ and $\operatorname{gcd}(a, b)=1$. For, we assume that $a>0, b \geq 0$ and $2 b=k a$ for some integer $k$, then $a^{2}-4 a b-4 b^{2}=\left(1-2 k-k^{2}\right) a^{2}=1$. It implies that $a=1$ and $k=0$ since $\operatorname{gcd}(a, b)=1$. That is $(a, b)=(1,0)$. When $(a, b)=(1,0)$, the $(a, b)$-type balancing number is nothing but the original balancing number. Thus, Theorem 1 actually says that there is no perfect power in the sequence of balancing numbers.

From the $y$-solutions of the Pell equation $x^{2}-8 y^{2}=1$, we see that $B_{m}^{(1,0)}=B_{m}$ is the coefficient of the irrational part in the expansion of $(3+\sqrt{8})^{m}$. If $m=2 k$, for some integer $k \geq 0$, we obtain that

$$
B_{2 k}=\sum_{j=0}^{k-1}\left(\begin{array}{c}
2 k \\
2 j+1
\end{array}\right) 3^{2 k-2 j-1} 8^{j}
$$

If $m=2 k+1$, we obtain that 


$$
B_{2 k+1}=\sum_{j=0}^{k}\left(\begin{array}{c}
2 k+1 \\
2 j+1
\end{array}\right) 3^{2 k-2 j} 8^{j}
$$

Similarly, the Lucas-balancing numbers are all $x$-solutions of the Pell equation $x^{2}-8 y^{2}=1$. So, we simply obtain that

$$
C_{2 k}=\sum_{j=0}^{k}\left(\begin{array}{l}
2 k \\
2 j
\end{array}\right) 3^{2 k-2 j} 8^{j}
$$

and

$$
C_{2 k+1}=\sum_{j=0}^{k}\left(\begin{array}{c}
2 k+1 \\
2 j
\end{array}\right) 3^{2 k-2 j+1} 8^{j}
$$

Corollary 1 . For any integer $k \geq 0$, we have

$$
\begin{aligned}
& B_{4 k+1} \equiv+1(\bmod 9), \quad B_{4 k+3} \equiv-1(\bmod 9) \\
& B_{2 k} \equiv-2 k(\bmod 8), \quad B_{2 k+1} \equiv 2 k+1(\bmod 8) ; \\
& C_{2 k} \equiv(-1)^{k}(\bmod 9), \quad C_{2 k+1} \equiv(-1)^{k+1} 3(k-1)(\bmod 9) \\
& C_{2 k} \equiv 1(\bmod 8), \quad C_{2 k+1} \equiv 3(\bmod 8)
\end{aligned}
$$

Of course, some explicit formulae of cobalancing and Lucas-cobalancing numbers, and some modular relations among them, can be obtained in a similar way. We leave it to the interested reader.

Author Contributions: Article by C.-L.C., C.Z. and K.Z. The authors contributed equally to this work. All authors have read and agreed to the published version of the manuscript.

Funding: This research was supported by Fuzhou University grant GXRC-18049:0030-510649, XRC18075:0030-510730, GXRC-19037:0030-510809, and Fujian Natural Science grant No. 2020J01498

Acknowledgments: The authors thank the anonymous reviewers for the comments concerning this paper. We found them to be helpful and constructive.

Conflicts of Interest: The authors declare no conflict of interest.

\section{References}

1. Behera, A.; Panda, G.K. On the square roots of triangular numbers. Fibonacci Q. 1999, 37, 98-105.

2. Panda, G.K. Sequence balancing and cobalancing numbers. Fibonacci Q. 2007, 45, 265-271.

3. Kovács, T.; Liptai, K.; Olajos, P. On $(a, b)$-balancing numbers. Publ. Math. Debr. 2010, 77, 485-498.

4. Subramaniam, K.B. Almost square triangular numbers. Fibonacci Q. 1999, 37, 194-197.

5. Newman, M.; Shanks, D.; Williams, H.C. Simple groups of square order and an interesting sequence of primes. Acta Arith. 1980, 38, 129-140. [CrossRef]

6. Barcucci, E.; Brunetti, S.; Del Lungo, A.; Del Ristoro, F. A combinatorial interpretation of the recurrence $f_{n+1}=6 f_{n}-f_{n-1}$. Discret. Math. 1998, 190, 235-240. [CrossRef] 